\title{
The definition of pregnancy-associated breast cancer is outdated and should no longer be used
}

The definition of pregnancy-associated breast cancer (PABC) is inconsistently given as either breast cancer diagnosed exclusively during pregnancy, or combined with cases diagnosed within 6 months to 1 year after the birth. ${ }^{1}$ Although pregnancy and the postpartum period are intertwined, evolving evidence supports considering breast cancer that occurs during pregnancy ( $\mathrm{PrBC}$ ) as a separate and distinct entity from breast cancer that occurs during the postpartum period (PPBC) - which, according to newer data, can extend to 5-10 years after the birth-because each type has unique biological attributes and prognosis.

$\operatorname{PrBC}$ is diagnosed during pregnancy and represents about $4 \%$ of cases of breast cancer in women younger than 45 years. $^{2}$ With an estimated incidence of 5.1 cases per 100000 livebirths in young women, breast cancer is one of the most common cancer types occurring during pregnancy. The existing literature gives a mixed view of whether a breast cancer diagnosis during pregnancy confers a worse prognosis than breast cancer not diagnosed during pregnancy. Although genetic differences and reduced overall survival in PrBC have been described, ${ }^{1}$ large cohort studies of PrBC find that clinical tumour characteristics and prognosis are indistinguishable from breast cancers occurring in young, nonpregnant women. ${ }^{3,4}$

PPBC occurs within 5-10 years after pregnancy and is estimated to represent $35-55 \%$ of all cases of breast cancer in women younger than 45 years. ${ }^{5} \mathrm{PPBC}$ is associated with worse survival rates and with a more than two times increased risk of metastasis than breast cancer diagnosed in young, premenopausal women during pregnancy or who have ever been pregnant (Figure, 1A). ${ }^{1,4}$ Poor prognosis persists after adjustment for several clinicopathological factors, including age at diagnosis, year of diagnosis, stage, grade, and hormone receptor status. ${ }^{6}$ Importantly, when the same data were grouped in the traditional PABC definition, as opposed to the biologically distinct proposed $\operatorname{PrBC}$ and PPBC definitions, this difference in outcomes was lost. ${ }^{2}$ Logically, if women at increased risk, such as women with PPBC, are included in a study as controls, the biological effect of previous childbirth is likely to go unidentified in any given analysis.

Since the conception of the definition of $\mathrm{PABC}$, more data on the cellular and molecular differences between PrBC and PPBC (including their respective tissue microenvironments [Figure, 1B]) have become available. During pregnancy, the mammary gland epithelium undergoes proliferation and differentiation in preparation for lactation. After childbirth and in the absence of lactation, or during weaning, the gland remodels to a state that is morphologically and functionally similar to the prepregnancy state, via a process called involution. It is hypothesised that involution, in the presence of subclinical disease, increases the metastatic potential of PPBC (Figure, 1B). ${ }^{7}$ Animal studies, supported by detailed studies of human breast involution, show wound healing-like alterations in the microenvironment of the involuting breast and an influx of immunosuppressive cells that promote the escape of tumour cells from the mammary gland. ${ }^{8,9}$ In patients with PPBC, patterns of altered immune infiltration, cytokine profiles, or both can persist in the primary tumour microenvironment for several years after delivery. ${ }^{10}$ As depicted in Figure 1B, the interaction between subclinical disease, wound healing-like stromal remodelling, and infiltration of tolerant immune cells could explain the worse prognosis of PPBC compared with PrBC, as well as the conflicting results observed in studies that consider both entities under a single definition.

The association between reproductive factors and breast cancer is complex, making it hard to differentiate between mechanisms related to nulliparity, uniparity, multiparity, age at first birth, lactation, involution, hereditary factors, and the breast tumour itself. Nevertheless, it is becoming clear that a distinction should be made between breast cancers that occur during pregnancy and those that 
occur within 5-10 years postpartum. In clinical practice, this difference is obvious: the treatment of $\operatorname{PrBC}$ is individualised according to gestational age and taking foetal safety into consideration, whereas treatment decisions for PPBC do not need to account for these concerns. Moreover, there is a need for a better understanding of the importance of parity status as an independent prognostic factor for worse outcomes in premenopausal breast cancer. Only a separate investigation of both entities will improve our understanding of the biology of breast cancer during pregnancy, lactation, involution, and thereafter, and help to decipher the pathways underlying differences in tumour biology. Therefore, we recommend that the term $P A B C$ is no longer used, allowing investigators to focus specifically on breast cancer during pregnancy (PrBC) or during the postpartum period (PPBC), which could ultimately lead to optimised therapeutic modalities, particularly for PPBC with a poor prognosis.

A

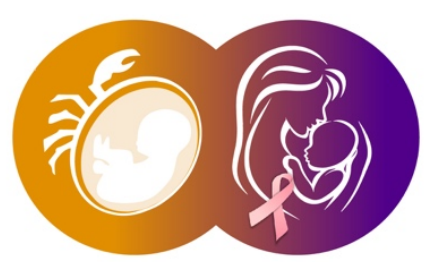

Pregnancy-associated breast cancer

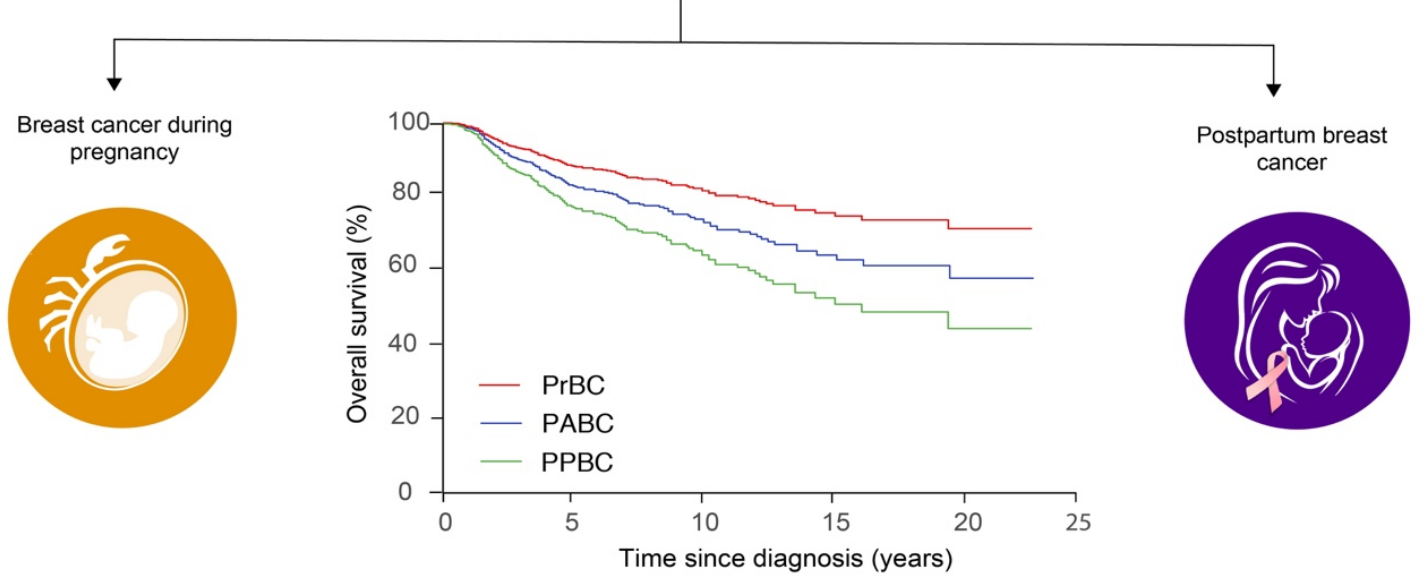

B

Mammary epithelium during pregnancy

Mammary epithelium during involution

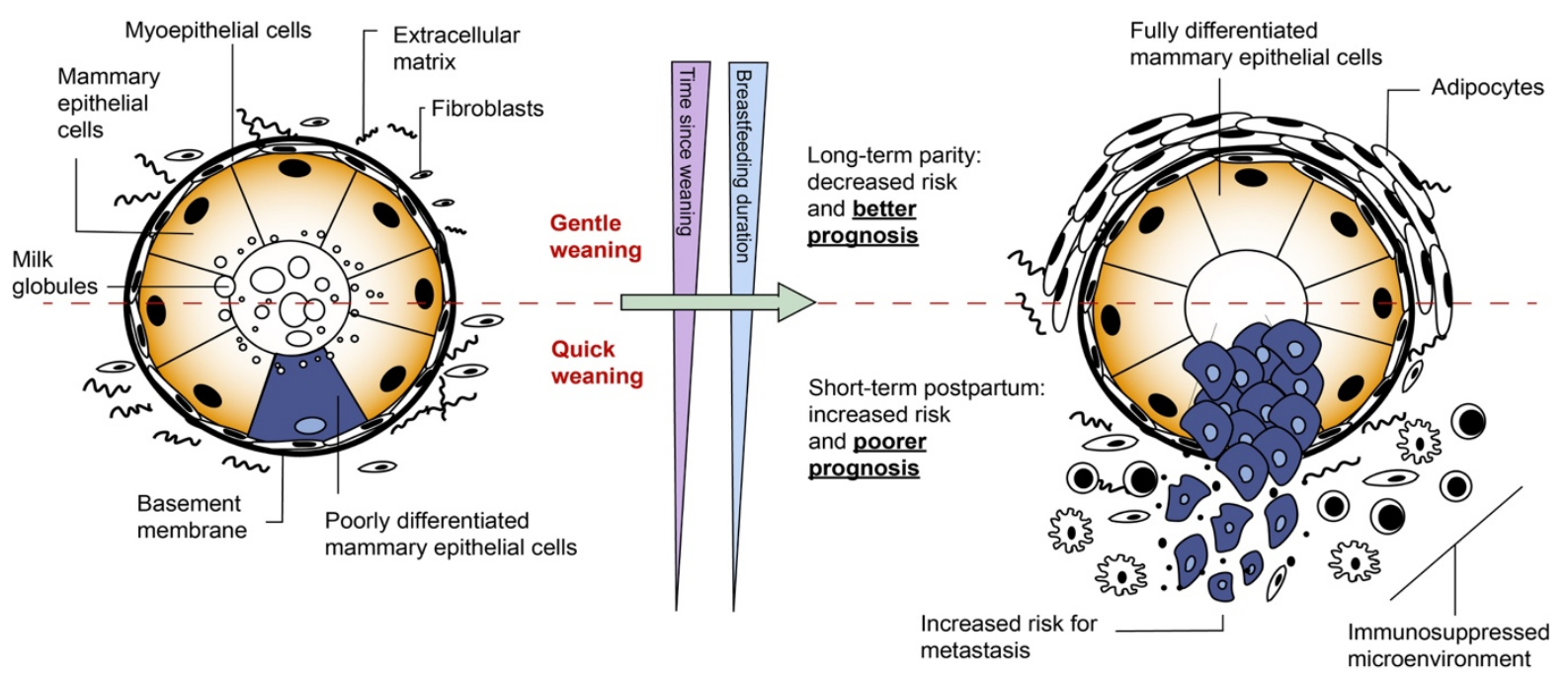

Figure 1. Schematic presentation of the cellular interactions causing the worse clinical outcome of postpartum breast cancer (A) The definition of PABC leads to conflicting clinical outcome data. When cases of PABC are differentiated between PrBC and PPBC, the worsened prognosis in this model is specifically true for PPBC. This distinction could explain the discrepancies in 
prognosis in published studies. (B) During pregnancy and lactation, proliferation and differentiation of the mammary gland that is purported to result in a long-term protective effect of pregnancy against breast cancer occurs. The short-term increased risk for PPBC is attributed to mammary gland involution, a process whereby mammary epithelial cells are subsequently removed by apoptosis to return the mammary gland to its prepregnant state (gentle weaning; upper panel). Involution shares numerous stromal attributes with protumour microenvironments, including immune suppression, which might lead to the formation of aggressive tumours with increased invasiveness (quick weaning; lower panel). PABC=pregnancy-associated breast cancer. $\mathrm{PPBC}=$ postpartum breast cancer. $\mathrm{PrBC}=$ breast cancer during pregnancy.

\section{References}

1. Lyons TR, Schedin PJ, Borges VF. Pregnancy and breast cancer: when they collide. J Mammary Gland Biol Neoplasia. Jun 2009;14(2):87-98.

2. Callihan EB, Gao D, Jindal S, et al. Postpartum diagnosis demonstrates a high risk for metastasis and merits an expanded definition of pregnancy-associated breast cancer. Breast Cancer Res Treat. Apr 2013;138(2):549-559.

3. Loibl S, Han SN, von Minckwitz G, et al. Treatment of breast cancer during pregnancy: an observational study. Lancet Oncol. Sep 2012;13(9):887-896.

4. Amant F, Von Minckwitz G, Han SN, et al. Prognosis of women with primary breast cancer diagnosed during pregnancy: Results from an international collaborative study. Journal of Clinical Oncology. 2013;31(20):2532-2539.

5. Borges $V$, Elder A, Lyons $T$. Deciphering pro-lymphangiogenic programs during mammary involution and postpartum breast cancer. Frontiers in Oncology. 2016-November-02 2016;6(227).

6. Shao C, Yu Z, Xiao J, et al. Prognosis of pregnancy-associated breast cancer: a meta-analysis. BMC Cancer. Aug 10 2020;20(1):746.

7. Schedin P. Pregnancy-associated breast cancer and metastasis. Nat Rev Cancer. Apr 2006;6(4):281-291.

8. Betts CB, Pennock ND, Caruso BP, et al. Mucosal immunity in the female murine mammary gland. Journal of Immunology. 2018;201(2):734-746.

9. Pennock ND, Martinson HA, Guo Q, et al. Ibuprofen supports macrophage differentiation, $T$ cell recruitment, and tumor suppression in a model of postpartum breast cancer. J Immunother Cancer. Oct 1 2018;6(1):98.

10. Lyons TR, O'Brien J, Borges VF, et al. Postpartum mammary gland involution drives progression of ductal carcinoma in situ through collagen and COX-2. Nat Med. Sep 2011;17(9):1109-1115. 